\title{
Editorial
}

\section{The Deadly Epidemic of Cardiovascular Disease}

\author{
Bhatt YKD \\ Shahid Gangalal National Heart Centre
}

In a recent interview by the famous TV personality and senior producer of Kantipur TV Suraj Singh Thakuri, I was suddenly asked "doc why does everyone talk of heart and not about other organs of the body? Nobody says I give my liver or kidney to you, why heart and why so much ado about this organ." Although this question appears to be just a means to initiate a conversation, it carries a lot of sense and delivers a concealed message.

Heart is the central organ of the body, supplying nutrients to all its parts; in other words, heart is responsible for keeping a person alive, healthy and functioning. Body will start malfunctioning even if the heart stops for a couple of seconds. Being one of the vital organs, it is the responsibility of an individual to keep heart healthy, hearty and functioning. Unfortunately, cardiovascular disease (CVD) has become a ubiquitous cause of morbidity and a leading contributor to mortality and morbidity in most countries. The emergence of the CVD epidemic in the developing countries during the past two to three decades has attracted very little comment and very little public health response.

It has been estimated that 5.3 million deaths attributable to CVD occurred in the developed countries in 1990, whereas the corresponding figure for the developing countries ranged between 8 to 9 million. Thus, in 1990 the developing countries contributed $68 \%$ of the total global deaths due to non communicable disease and $63 \%$ of world mortality due to CV diseases.

\section{Historic experience and observation}

It has been the historical experience of the developed Countries that the CVD epidemic usually commences in members of the higher social classes, who are the first to change from a low-risk to a high-risk lifestyle, which is characterized by diets rich in fat and calories, sedentariness, and smoking. Later, the risk permeates across the social spectrum, affecting all classes. The higher-social classes are, again, the first to respond to the knowledge of risk factors and the message of prevention. They are the ones who will begin the preventive measures and respond to it early and avail the benefit of preventive programme. Many a times, generations are lost in this realization. In the developed countries, the Cardiovascular Diseases have risen and have started to decline gradually. The rise and recent decline of the CVD epidemic in the developed countries have been well documented.

The identification of major risk factors through populationbased studies and effective control strategies combining community education and targeted management of high risk individuals have contributed to the fall in CVD mortality rates (inclusive of coronary and stroke deaths) that has been observed in almost all industrialized countries. It has been estimated that during the period 1965 to 1990, CVD related mortality fell by $50 \%$ in Australia, Canada, France, and the United States and by $60 \%$ in Japan. Other parts of Western Europe reported more modest declines (20\% to $25 \%)$. The decline in stroke mortality has been more marked compared with the decline in coronary mortality. In the United States, the decline in stroke mortality commenced nearly two decades earlier than the decline in coronary mortality and maintained a sharper rate of decline. During the period 1979 to 1989 , the age-adjusted mortality from stroke declined in that country by about one third, whereas the corresponding decline in coronary mortality was $22 \%$.

\section{Lifestyle Changes}

Adverse lifestyle changes accompanying industrialization and urbanization has contributed to higher prevalence of coronary heart disease (CHD) and Cardiovascular disease. Lack of exercise, adverse food habits have definitely led to higher levels of body mass index, blood pressure, fasting blood lipids (total cholesterol, ratio of cholesterol to HDL cholesterol, triglycerides), and diabetes. All of these being risk factors for CVD. The natural physical exercise associated with rural lifestyle is lost as a society develops into an urban one. Studies have clearly revealed that average body mass index of rural population is much lower than then the urban counterpart.

\section{Tobacco Trends}

The increasing use of tobacco in a number of developing countries will also translate into higher mortality rates of CVD, lung cancer, and other tobacco-related diseases. The rising tobacco consumption patterns in most developing 
countries contrast sharply with the overall decline in the industrialized nations. Recent projections from the World Health

Organization suggest that, by the year 2020, tobacco will become the largest single cause of death, accounting for $12.3 \%$ of global deaths. India, China, and countries in the Middle

Eastern area will by then have tobacco contributing to $12 \%$ of all deaths. In India alone, the tobacco attributable toll will rise from $1.4 \%$ in 1990 to $13.3 \%$ in 2020.

A large component of this will be in the form of cardiovascular deaths. I am sure this is applicable to our country also. Various anecdotal reports have shown a smoking prevalence of $16-74 \%$ in different parts of Nepal. Tobacco is the leading avoidable cause of death worldwide, and its rising consumption in the developing countries warrants early and effective public health response. In a small study in referral hospital in Kathmandu it was seen that $55.5 \%$ of the individuals who had Myocardial Infarction were smokers.

\section{Coronary artery disease}

In United States, there are one million deaths each year and half of it is due to Coronary Artery Disease (CAD). 1.5 million suffer from myocardial Infarction each year.

Coronary artery disease is not only a deadly disease, it may also be a devastating disease as it has a tendency to strike at the peak of ones working carrier. $45 \%$ of the Myocardial Infarctions (MI) occur below the age of 65 years. $2 / 3$ rd of the people who have $\mathrm{MI}$ survive and $1 / 3$ do not. Among the $2 / 3^{\text {rd }}$ who survive, $2 / 3$ do not make a full recovery. It has been seen that $37 \%$ of American males and $29 \%$ females who die of Ml are below the age of 55 .

\section{Contributors of fall in CVD in west}

As previously stated CVD is on the fall in the western world and is affecting age group which is much elder to those in the developing world. The incidence of CVD deaths below the age of 70 years is $46.7 \%$ in the developing world as compared to $26.5 \%$ in the developed world.

The major contributor of fall in CVD in the west is the effective control strategies.

At first the risk factors of CV diseases and their prevalence in the country was estimated and evaluated. Studies like the Framingham study well established the relationship between various groups of age, sex and the risk factors. This clearly brought forward the average risk of an individual to various CV diseases. As this education was propagated, the educated higher socioeconomic society, responded dramatically. Community education along with focus on the high risk individuals further added in the success in the fall in CV diseases.
Coronary Artery disease (CAD) death rate fell $54 \%$ from 1963 to 1990 in US. Between 1982 to 1992, there was 31\% decline in Myocardial infarction deaths. Similarly there has been a decline in smoking by $37 \%$ since 1965 . The annual death rate from Hypertension decreased from 56/100,000 in 1965 to $6.5 / 100,000$ in 1991 . Average plasma Cholesterol has declined from 220 to $205 \mathrm{mg} \%$ in age 20 to $74 \mathrm{yrs}$ between 1960 and 1991.

\section{Tragedy with developing countries}

The tragedy with the developing countries is very tragic. According to World Health Organization, $30 \%$ of global death in 1998 was due to Cardiovascular Disease, 15.3 million lives were lost that year. It is interesting to note that low and middle income countries contributed $78 \%$ of the deaths demonstrating the gravity of the problem in these regions.

It has been estimated that the developing countries contribute $84 \%$ of the world's population and $93 \%$ worldwide burden of disease. On the other hand the developing countries generate only $18 \%$ of global economy and account for only $11 \%$ of global health spending. The projected rise in CVD mortality in India and possibly in the subcontinent is 103\% in men and 90\% in women from 1985 to 2015 . By 2015 , CVD would account for $34 \%$ of all male and $32 \%$ of all female deaths.

The estimated rise in Coronary Artery disease by 2020 in the developing countries is $137 \%$ and $120 \%$ in men and women respectively compared to $48 \%$ and $29 \%$ in the developed countries. The stroke burden would rise by $124 \%$ and $107 \%$ in men and women compared to $56 \%$ and $28 \%$ in developed countries. As can be visualized, this healthcare and economic burden is beyond the scope of most developing countries.

\section{CVD scenario in Nepal}

In Nepal the incidence and prevalence of CVD has not been well defined. There are few studies which have shown statistics in certain group of people or in a certain region of the country but the national data is no where in the horizon. It has been seen that the prevalence of Hypertension is around 20\%, Rheumatic Heart disease is $1.1 / 1000$, Metabolic syndrome $12.4 \%$ and Coronary artery disease around 5\% (in Kathmandu).

Thus, the new century dawns on a period of challenge an opportunity for the developing countries as they embark on their efforts to quell the emerging epidemic of CVD. National and international efforts must be coordinated to recognize this epidemic and respond without delay.

As an educated responsible individual of the country, it is not only our duty to be aware of the cardiovascular risk factors and be the early responders to it, but also to promulgate the knowledge of CVD and its prevention in the society. 